\title{
Analytical lifecycle management oriented development and validation of a new stability- indicating ultra-fast liquid chromatographic method: case study of Glycopyrrolate
}

\author{
Sagar Suman Panda ${ }^{(i *}$ B. V. V. Ravi kumar, Suchismita Swain \\ Department of Pharmaceutical Analysis \& Quality Assurance, Roland Institute of Pharmaceutical \\ Sciences (Affiliated to Biju Patnaik University of Technology, Odisha), Berhampur, Odisha, India
}

\begin{abstract}
A new stability-indicating liquid chromatographic method was developed and validated for the estimation of glycopyrrolate in pharmaceutical formulations. A contemporary approach to analytical life-cycle management was followed to develop a robust and reliable chromatographic method. Scouted method variables such as \% methanol, the strength of tetra butyl ammonium hydrogen sulfate and mobile phase flow rate were optimized using the design of experiment approach and their effect on critical quality attributes was studied. The critical quality attributes viz. retention time, theoretical plate count and symmetry factor were highly influenced by the three critical method variables. Optimum chromatography was attained on a C-18 column with a mobile phase methanol: 10 $\mathrm{mM}$ tetra butyl ammonium hydrogen sulfate $(80: 20, \mathrm{v} / \mathrm{v})$ flowing at $1.0 \mathrm{~mL} \cdot \mathrm{min}^{-1}$. Chromatographic method specificity was ensured by degrading the drug forcefully. Validation studies postulated method acceptability and suitability for estimating glycopyrrolate in both bulk as well as injection formulation. Results for parameters viz. linearity $\left(5-250 \mu \mathrm{g} \cdot \mathrm{mL}^{-1}\right)$, accuracy $(>99 \%)$ and precision $(<2 \%)$ advocated method reliability. Overall the method was reliable and of optimum quality and, possess the potential of application in routine and bio-analytical purposes.
\end{abstract}

Keywords: Glycopyrrolate. Stability-indicating. Liquid chromatography. Life cycle management. Validation.

\section{INTRODUCTION}

Analytical lifecycle management (ALM) is a novel approach which derives its basic principle from the combination of ICH guidelines Q8, Q9 and Q10 (USP, 2013). It has several benefits over the traditional approach as it integrates validation, transfer and verification of procedure (Parr, Schmidt, 2018). This approach is divided into three stages starting with procedure design, which includes defining the analytical target profile (ATP) and critical quality attributes (CQAs). Once the ATP and CQAs are defined, the quality risk management

*Correspondence: S. S. Panda, Department of Pharmaceutical Analysis and Quality Assurance, Roland Institute of Pharmaceutical Sciences, Ambapua, Berhampur-760010. Odisha - India. E-mail:sagarguddu2002@ gmail.com
(QRM) tools like fish-bone diagram, control-noiseexperimental $(\mathrm{C}-\mathrm{N}-\mathrm{X})$ approaches are best utilized to identify the critical method variables (CMVs) demanding further studies. The identified variables are investigated using the design of experiments (DoE) to minimize the risks and optimize the experimental conditions. The second stage is known as procedure performance qualification which includes experimentation based on optimized conditions and suitable analytical control strategies are derived. In the conclusive stage i.e. procedure verification the compliance with analytical control strategy is monitored continuously to improve the method performance. Considering the advantages over the traditional approach and lack of analytical methods with the ALM framework, the authors decided to implement the same for the present research. 
Another important aspect of developing a quality analytical method is selecting the proper analytical technique. Ultrafast liquid chromatography (UFLC) is a better option for rapid chromatographic studies and is widely used by analysts throughout the world (Panda et al., 2013a; Panda et al., 2013b; Panda et al., 2014). Low mobile phase usage and faster reproducible analysis are the two advantages of UFLC over conventional HPLC, advocating its suitability for use in routine applications.

In the present study glycopyrrolate(GLP), 3 - $\{[$ cyclopentyl(hydroxy) phenylacetyl] oxy $\}-1,1-$ dimethylpyrrolidin-1-ium bromide (Figure 1) is an anti-cholinergic antispasmodic drug and also used in treating chronic severe drooling (O'Neil, 2006; Evatt, 2011). Based on literature review it was found that few HPLC methods are reported for analysis of GLP in various sample matrixes (Nebiu et al., 2007; Rumpler, Sams, Colahan, 2011; Gandala, Pabba, Akula, 2011; Kusuma, Rao, Rameshraju, 2016; Misra, Arora, 2016). However, the reported methods possessed different drawbacks like using complex and corrosive mobile phases, need of column oven, high mobile phase flow rate, lower sensitivity, lack of reliability etc. Moreover, none of the reported methods is ALM supported ensuring the reliability of data obtained. Hence, lack of reliability, as well as robustness, were the two critical aspects basing upon which the authors attempted to develop a new reversed-phase UFLC method for estimation of GLP present in bulk as well as injections. Further, method validation studies were carried out as per ICH Q2 (R1) guidance (ICH, 2005). Forced degradation revealed the stability nature of GLP. Injection formulation of GLP containing $0.2 \mathrm{mg} \cdot \mathrm{mL}^{-1}$ of the drug was analyzed by the developed method.

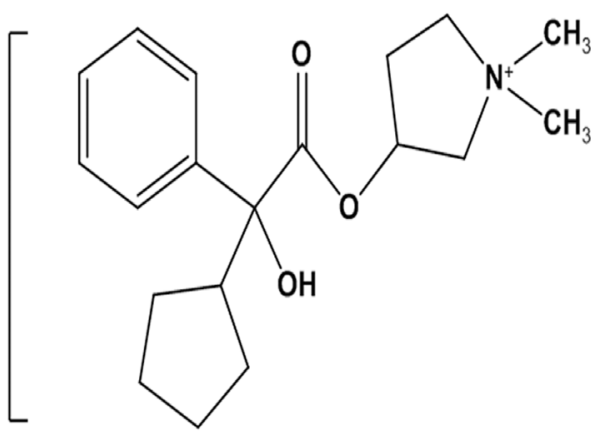

7

FIGURE 1 - Chemical structure of GLP.

\section{MATERIAL AND METHODS}

\section{Material}

Standard GLP (purity $>98 \%$ ) was kindly provided by Roland Institute of Pharmaceutical Sciences, Berhampur. Melting point depression of GLP was measured by Shimadzu DSC-60 (Differential Scanning Calorimeter) Thermal Analyzer. Purity assessment of organic and pharmaceutical compounds by measuring the melting point employing DSC is an age-old established technique(Brennan et.al., 1984; Deangelis, Papariello, 1968; van Dooren, Muller, 1984).The melting point of GLP was found to be $195.36{ }^{\circ} \mathrm{C}$ which is within the prescribed values of $193-198{ }^{\circ} \mathrm{C}$ (US FDA, 2010).The compendial specifications for GLP consider the ICH Q3B (R2) guidance and establish the overall purity of drug being not less than $98 \%$ with an individual impurity threshold of not more than $0.15 \%$ for the impurities, except the erythro isomer where the threshold is up to $0.4 \%$ (USP, 2017). HPLC grade methanol and analytical reagent grade tetra butyl ammonium hydrogen sulfate (TBAHS), hydrochloric acid, sodium hydroxide, hydrogen peroxide were used for the purpose. HPLC grade water prepared by TKA GenPure Ultra-Purification System, Germany was used as the aqueous phase. The commercial injection containing $0.2 \mathrm{mg} . \mathrm{mL}^{-1}$ of GLP in water for injection along with preservative benzyl alcohol $(0.9 \%)$ and hydrochloric acid and/or sodium hydroxide for $\mathrm{pH}$ maintenance was analyzed by the UFLC method.

\section{Instrumentation and chromatographic condition}

A SHIMADZU Prominence Series UFLC equipped with binary pumps and a PDA detector was used for the purpose. A reversed-phase column $(250 \times 4.6 \mathrm{~mm}, 5 \mu \mathrm{m})$ was used for chromatography. The mobile phase contained methanol and $10 \mathrm{mM}$ TBAHS in a composition of $80: 20 \%, \mathrm{v} / \mathrm{v}$ with a flow rate of $1 \mathrm{~mL} \cdot \mathrm{min}^{-1}$, and detection at $204 \mathrm{~nm}$. Prior use both the mobile phase components were sonicated for degassing.

\section{Procedure Design}

Setting ATP and CQAs

The ATP for the present study was to develop and validate a rapid, robust and reliable chromatographic method for estimation of GLP in bulk and injection 
formulation. Based on the ATP a UFLC was selected for the purpose. The selected CQAs were retention time, plate number and symmetry factor.

\section{Risk management}

Risk assessment is a constitutive part of effective risk minimization process. Ishikawa fish-bone diagram acts as a preliminary tool for risk assessment and addresses all potential method variables that can affect the CQAs adversely. From the various variables, a critical few are selected after being subjected to a C-N-X analysis. Method variables with scores higher than 200 were selected as the critical few and were further subjected to DoE investigation. Three variables viz. \% methanol, the strength of TBAHS solution and flow rate of the mobile phase were identified as CMVs for DoE based investigation.

\section{Design of experiment and method optimization}

Based on the number of variables to be studied it was found suitable to choose a Box-Behnken design (BBD) for the robustness investigation and optimization study. Minimal 15 runs were performed in a randomized manner having 3 centre points. The obtained experimental data was analyzed mathematically to study any possible interaction effect among the variables. Further statistical data analysis was carried out to evaluate the aptness of the study model. Various model parameters along with polynomial equations, 2-dimensional and 3-dimensional plots were assessed for checking the model suitability. Based on the results of the above assessment analytical control space was developed for procedure performance qualification purpose.

\section{Procedure Performance Qualification}

\section{Analytical control strategy}

Control strategies were developed based on the optimized method conditions and control charts were prepared for the CQAs over a period of 6 days $(n=3)$. It helped to derive robust working limits for the method variables to obtain desired CQAs.

\section{Procedure Verification}

It consisted of continuous monitoring of the newly optimized analytical method in order to reduce any incident of risks arising from time to time and to improve the method performance in terms of specified CQAs.

\section{Method Validation}

\section{Specificity}

Specificity capability of this current method was confirmed by forcefully degrading GLP in an ICH compliant framework. The ICH guidance advocates for any suitable set of experimental conditions which can result in degradation of the analyte to a considerable level and generate degradation products, if any. Hence, to attain the best of both of these objectives, stress conditions were developed utilizing minimal time and chemicals. Stress conditions like acid, alkali, peroxide, temperature and UV light helped in the degradation of GLP. An aliquot of $400 \mu \mathrm{L}$ of GLP was taken in five different $5 \mathrm{~mL}$ volumetric flasks. GLP was exposed to $500 \mu \mathrm{L}$ of $0.1 \mathrm{M}$ $\mathrm{HCl}, 0.1 \mathrm{M} \mathrm{NaOH}, 3 \% \mathrm{H}_{2} \mathrm{O}_{2}, 80{ }^{\circ} \mathrm{C}$ and UV light (365 nm) for a specified time period (30min for all the conditions except photolysis where the exposure time was up to $3 \mathrm{~h}$ ). Afterwards, the acid and alkali stressed samples were neutralized using respective counterparts, and the other samples were diluted to $5 \mathrm{~mL}$ with mobile phase. The amount of drug degraded was calculated by comparing the results with the untreated drug solution. GLP at $80 \mu \mathrm{g} \cdot \mathrm{mL}^{-1}$ was chosen for the forced degradation study purpose.

\section{Preparation of Calibration Curve}

Around 10mg of accurately weighed GLP was dissolved in mobile phase placed in a $10 \mathrm{~mL}$ volumetric flask. Further, this solution was used to prepare working standard solutions within 5-250 $\mu \mathrm{g} \cdot \mathrm{mL}^{-1}$ of GLP. The average peak areas $(n=3)$ for each concentration were used to prepare the curve.

\section{Accuracy}

A fixed concentration $\left(80 \mu \mathrm{g} \cdot \mathrm{mL}^{-1}\right)$ solution of GLP present in injection solution was chosen and was spiked with $80 \%, 100 \%$ and, $120 \%$ of standard GLP. Recovery of standard GLP was indicative of method trueness.

\section{Precision}

Method precision was assessed in terms of repeatability, intermediate and instrument precision at 
$80 \mu \mathrm{g} \cdot \mathrm{mL}^{-1}$. The precision of the UFLC was determined based on hexaplicate injection of aforementioned concentration of GLP, whereas the repeatability and intermediate precision were determined from injection of six separately prepared solution of the analyte at the same concentration. A \% relative standard deviation $(\% \mathrm{RSD})$ value less than 2.0 was the acceptance limit.

\section{Sensitivity}

Signals of the lowest concentration of samples were measured and compared to that of blank to establish the effective concentrations of the analyte as the limit of detection (Signal/Noise=3) and quantitation (Signal/ Noise $=10$ ). Analyte concentrations producing the desired signal-to-noise ratio were designated as LOD and LOQ, respectively.

\section{Assay of a commercial formulation}

An injection volume equivalent to $5 \mathrm{mg}$ of GLP was taken and ultrasonicated for $30 \mathrm{~min}$ in the presence of mobile phase. Once the final volume was made up this solution was filtered using a membrane filter $(0.45 \mu)$. Thereafter, it was utilized to prepare a sample solution for LC analysis. Further, solutions of formulation components such as hydrochloric acid and sodium hydroxide mixture (acts as $\mathrm{pH}$ balancing agent) and benzyl alcohol (preservative) were also prepared separately and injected onto the UFLC to assess any possible interference at analytes retention time.

\section{RESULTS AND DISCUSSION}

\section{Preliminary method development using a lifecycle management approach}

According to the physicochemical property of analyte, a reversed-phase LC method was found befitting for chromatography of GLP. GLP is a highly polar compound with a greater tendency for early chromatographic elution. This typical problem was overcome by choosing a superior ion-pair reagent like tetra butyl ammonium hydrogen sulfate (TBAHS) which has the capability of enhancing method selectivity as well as the solubility of GLP and also may reduce adsorptive interaction on the stationary phase. The literature revealed that other researchers have used complex and corrosive mobile phases, column oven, and high mobile phase flow rate. Moreover, the methods were less sensitive and lacked reliability. Addressing these demerits in the present paper $10 \mathrm{mM}$ TBAHS solution was used as the aqueous phase avoiding any blockage issue due to high amounts of buffer salts. Further, none of the reported methods utilized $10 \mathrm{mM}$ TBAHS solution as the mobile phase for estimating GLP. Hence, considering the above facts TBAHS solution was chosen as the aqueous phase. The typical mobile phase constituted of methanol: 10 $\mathrm{mM}$ TBAHS. Various compositions of mobile phase and flow rates such as $1.0 \mathrm{~mL}, 1.1 \mathrm{~mL}$ and $1.2 \mathrm{~mL}$ were tested to check the response of GLP at $204 \mathrm{~nm}$. A composition of methanol: $10 \mathrm{mM}$ TBAHS at 80:20, v/v flowing at 1.0 $\mathrm{mL} \cdot \mathrm{min}^{-1}$ gave optimum peak performance.GLP eluted at 3.134minwith a run time of 7 with Further, considering the above conditions as initial method development start point, life cycle management approach was followed for establishing a reliable stability-indicating analytical method for estimation of GLP in pharmaceuticals.

With the intent of accomplishing ATP preliminary risk assessment and its minimization was carried out. The typical fish-bone based causal-effect relationship diagram (Figure 2) depicted numerous method variables with a possible threat to method performance quality. Out of the different risk, causes were assessed for the level of risk using a traffic light risk analysis matrix. In the mentioned matrix variables were designated to high, medium and low risk through colours viz. red, yellow and green, respectively (Table I). Certain method variables were found to possess a possible risk to the method performance i.e. CQAs. For enhanced discernment of the criticality of these variables a controlled noise experimental (C-N-X) approach was initiated and the results are displayed in Table II. This approach concluded that the method variables such as $\%$ methanol composition, the strength of TBAHS solution and flow rate had the highest risk potential based on their CNX score. Further, these three variables were named as CMVs and subjected to DoE investigation in order to enhance method robustness, optimize the method and minimize the incidence of any risks to method performance.

\section{Design of experiments based investigation}

The BBD domain proved to be worthy, as it unearthed the influence of CMVs on the CQAs. Experiments performed on randomized manner provided bias-free results (Table III). 


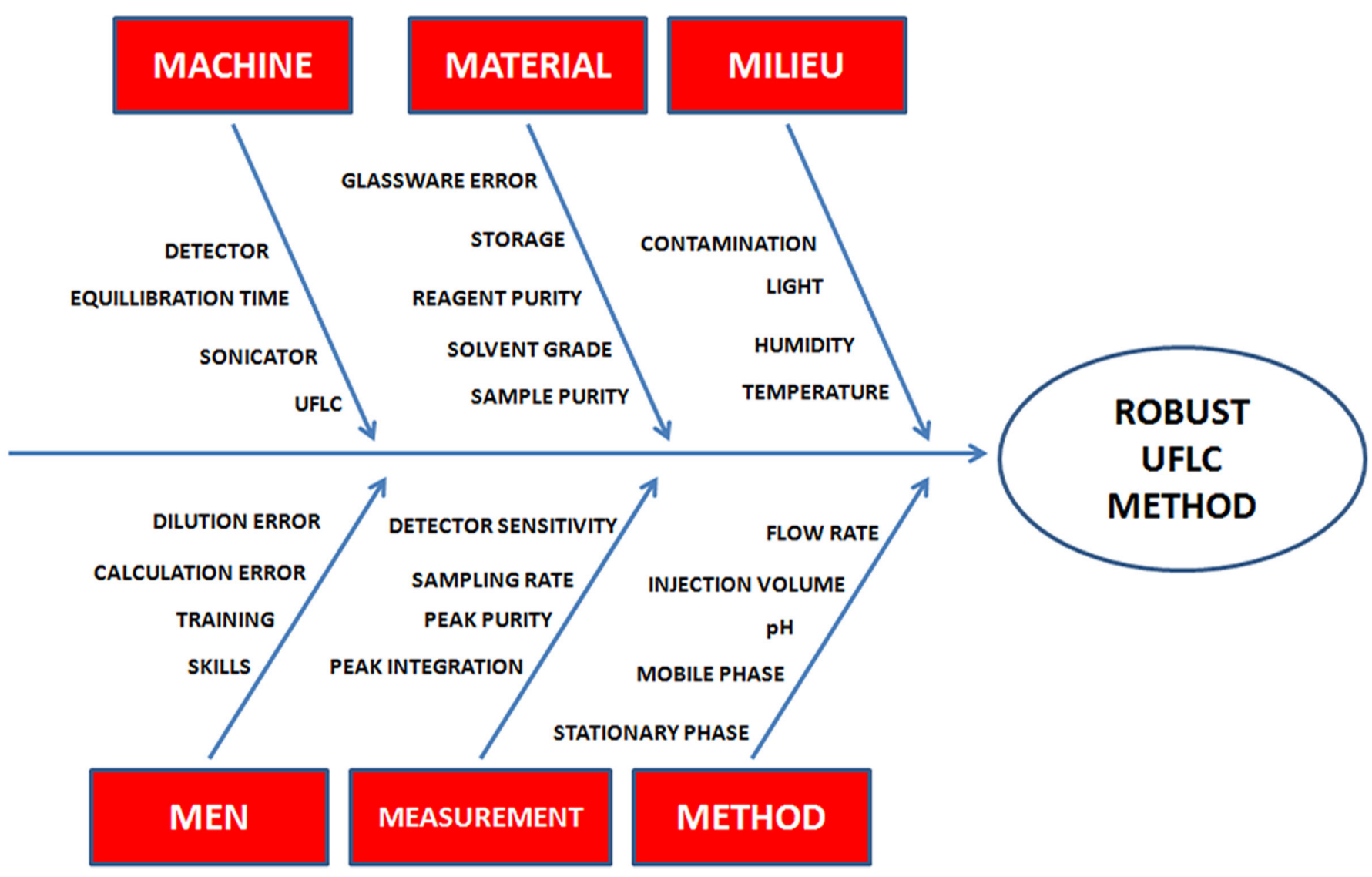

FIGURE 2 - Fish-bone diagram explaining causal-effect relationship between variables and method intent.

The optimization data was subjected to appropriate mathematical models for analysis. Polynomial equations (Eq.1, 2 and 3) consisting of model terms for both main effects and interaction effects were generated for the CQAs. It helped to unearth the connection among the CMVs and CQAs, for a sound understanding of the various effects. Synergistic and antagonistic effects were sorted from the polynomial equations based on positive and negative signs of each coefficient.

Assessment of ANOVA $(\mathrm{P}<0.05)$ along with satisfactory values of $\mathrm{r}^{2}\left(\mathrm{r}^{2}>0.9\right)$ advocated for the appropriacy of the selected mathematical model for obtaining optimum values of CQAs. Significant nature of the studied models was established by evaluating the predicted F-values obtained $(4435.78,282.3$, and 79.17 for retention time, theoretical plate count and symmetry factor, respectively). Model appropriacy for design space analysis was further ascertained by obtaining adequate precision (signal-to-noise ratio) values greater than 4.0 for all the three CQAs.

Response surface evaluation was performed employing 3-D plots (Figure 3-(A-I). Figure 3(A) depicts a slight decrease in retention time at high levels of $\%$ methanol and strength of TBAHS. A very similar interpretation was noticed for the CMVs \% methanol and flow rate (Figure 3(B)). A typical "hat" shaped response surface was obtained depicting complex interaction among the CMVs \% methanol and strength of TBAHS (Figure 3(C)). Increased retention times were seen at low levels of both the CMVs. However, the retention of GLP decreased gradually with the increasing flow of mobile phase and with low levels of strength of TBAHS. 
TABLE I - Traffic light risk analysis matrix

\begin{tabular}{|c|c|c|c|}
\hline Symmetry factor & Theoretical Plate Count & Retention Time & CQAs \\
\hline HIGH & HIGH & HIGH & Mobile Phase \\
\hline HIGH & HIGH & HIGH & Strength of TBAHS \\
\hline LOW & LOW & MEDIUM & Injection volume \\
\hline LOW & MEDIUM & HIGH & Solvent grade \\
\hline LOW & LOW & HIGH & Sample Purity \\
\hline LOW & LOW & MEDIUM & Reagent Purity \\
\hline LOW & MEDIUM & LOW & Humidity \\
\hline LOW & LOW & LOW & Temperature \\
\hline MEDIUM & LOW & LOW & Peak integration \\
\hline MEDIUM & MEDIUM & LOW & Peak Purity \\
\hline MEDIUM & MEDIUM & MEDIUM & UFLC \\
\hline HIGH & HIGH & HIGH & Flow rate \\
\hline LOW & LOW & LOW & Sonicator \\
\hline MEDIUM & MEDIUM & MEDIUM & Calculation Error \\
\hline LOW & MEDIUM & LOW & Dilution Error \\
\hline LOW & MEDIUM & LOW & Glassware error \\
\hline MEDIUM & LOW & MEDIUM & Equilibration Time \\
\hline LOW & MEDIUM & HIGH & Stationary Phase \\
\hline
\end{tabular}


TABLE II - Typical control-noise-experimental (C-N-X) based identification of CMVs

\begin{tabular}{|c|c|c|c|c|c|c|}
\hline \multirow[b]{2}{*}{ Method Variables } & \multicolumn{3}{|c|}{ Risk Level* on CQAs } & \multirow[b]{2}{*}{ Score } & \multirow[b]{2}{*}{$\mathbf{C}, \mathbf{N}, \mathbf{X}$} & \multirow[b]{2}{*}{ Action Plan } \\
\hline & $\begin{array}{c}\text { Retention } \\
\text { time }\end{array}$ & $\begin{array}{l}\text { Theoretical } \\
\text { Plate Count }\end{array}$ & $\begin{array}{c}\text { Symmetry } \\
\text { factor }\end{array}$ & & & \\
\hline$\%$ methanol & 10 & 10 & 10 & 300 & $\mathrm{X}$ & DoE \\
\hline Flow rate & 10 & 8 & 7 & 250 & $\mathrm{X}$ & DoE \\
\hline Sample Purity & 6 & 3 & 3 & 120 & $\mathrm{~N}$ & Quality Assessed \\
\hline UFLC & 3 & 3 & 2 & 80 & $\mathrm{C}$ & Controlled \\
\hline Calculation error & 2 & 2 & 2 & 60 & $\mathrm{~N}$ & Careful Measurement \\
\hline
\end{tabular}

$*$ Risk Level: 1-Negligible, 5 -Low, 10-High; Final Score $=\left(\right.$ Risk Level of $\left.1^{\text {st }} C Q A \times 10\right)+\left(\right.$ Risk Level of $\left.2^{\text {nd }} C Q A \times 10\right)+($ Risk Level of $3^{\text {rd }} \mathrm{CQA} \times 10$ ) 
Table III - Results for robustness-cum-optimization study by Box-Behnken experimental domain

\begin{tabular}{lcccccc}
\hline Run & \% Methanol & $\begin{array}{c}\text { Strength of } \\
\text { TBAHS (mM) }\end{array}$ & $\begin{array}{c}\text { Flow rate } \\
\left(\mathbf{m L} \cdot \mathbf{m i n}^{-1}\right)\end{array}$ & $\begin{array}{c}\text { Retention } \\
\text { Time(min) }\end{array}$ & $\begin{array}{c}\text { Theoretical } \\
\text { Plate Count }\end{array}$ & $\begin{array}{c}\text { Symmetry } \\
\text { factor }\end{array}$ \\
\hline 1 & 80 & 10 & 1.0 & 3.157 & 2732 & 1.628 \\
2 & 82 & 10 & 0.9 & 3.231 & 2576 & 1.546 \\
3 & 80 & 10 & 1.0 & 3.155 & 2739 & 1.621 \\
4 & 82 & 9.5 & 1.0 & 3.086 & 2387 & 1.492 \\
5 & 80 & 9.5 & 0.9 & 3.41 & 2643 & 1.621 \\
6 & 80 & 1.1 & 2.933 & 2584 & 1.623 \\
7 & 9.5 & 1.1 & 3.182 & 2625 & 1.601 \\
8 & 78 & 10 & 1.0 & 3.185 & 2678 & 1.78 \\
9 & 78 & 9.5 & 1.0 & 3.155 & 2742 & 1.624 \\
10 & 80 & 10 & 1.0 & 3.218 & 2599 & 1.574 \\
11 & 78 & 10.5 & 1.0 & 2.948 & 2487 & 1.651 \\
12 & 82 & 10.5 & 1.1 & 3.111 & 2523 & 1.542 \\
13 & 80 & 10.5 & 1.1 & 2.824 & 2501 & 1.492 \\
14 & 82 & 10 & 0.9 & 3.114 & 2727 & 1.611 \\
\hline 5 & 80 & 10 & 0.9 & 3.251 & 2805 & 1.656 \\
\hline
\end{tabular}

\section{Coded}

\section{Levels}

\begin{tabular}{llcl} 
Low & 78 & 9.5 & 0.9 \\
Mid & 80 & 10 & 1.0 \\
High & 82 & 10.5 & 1.1 \\
\hline
\end{tabular}

Where $\mathrm{A}=$ Methanol $(\%), \mathrm{B}=$ Strength of TBAHS $(\mathrm{mM})$ and $\mathrm{C}=$ Flow rate $\left(\mathrm{mL} \cdot \mathrm{min}^{-1}\right)$ 

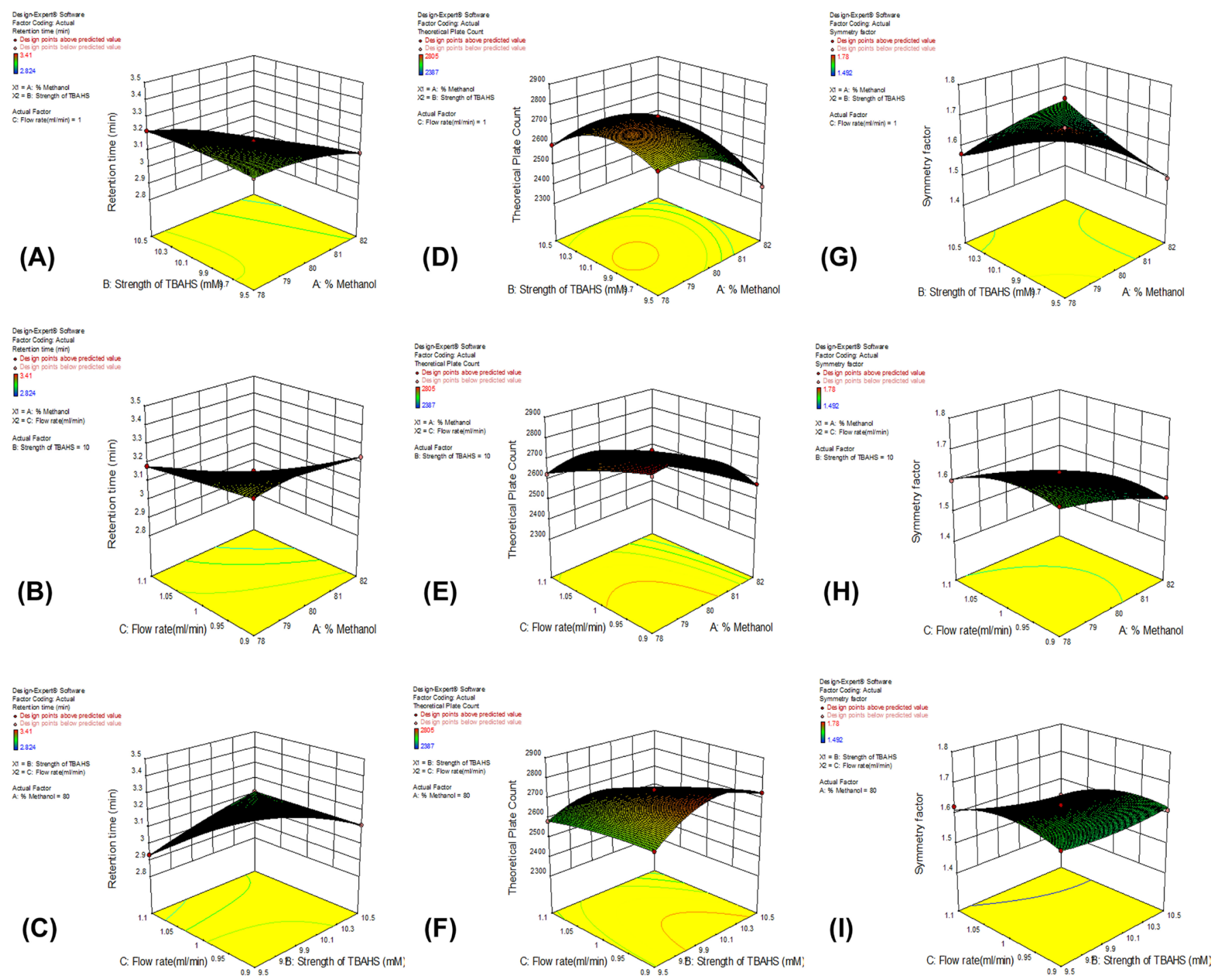

FIGURE 3 - Three dimensional response surfaces obtained for CQAs viz. (A-C) retention time, (D-F) theoretical plate count and (G-I) symmetry factor using experimental design approach.

A "maxima" system depicting maximum response was obtained around the lower-mid level of $\%$ methanol and mid-levels of the strength of TBAHS. The theoretical plate count was found to decrease with increasing values of methanol content at all levels of strength of TBAHS (Figure 3(D)). The theoretical plate values were found decreasing in a linear pattern at low and high levels of methanol content and around all the levels of strength of TBAHS. However, an increase in theoretical plates around mid-levels of \% methanol was noticed (Figure 3(E)).A curvilinear response surface was obtained for theoretical plates with increasing strength of TBAHS throughout all levels of flow rate (Figure 3(F)).

A typical "hat" shaped response surface was obtained depicting complex interaction among the CMVs \% methanol and strength of TBAHS (Figure $3(\mathrm{G})$ ). Increased tailing was observed at low levels of both the CMVs. However, the tailing of GLP decreased gradually with increasing methanol content and at low 
levels of strength of TBAHS. No significant change in tailing was noticed with a change in \% methanol and flow rate of mobile phase (Figure 3(H)). Tailing was found to decrease to a small extent with higher strength of TBAHS and high levels of flow rate (Figure 3(I)). Analogous information was drawn by interpreting the 2-dimensional contours (not shown in figures) for all the respective CMVs.

Response sensitiveness was evaluated by perturbation plots (not shown in figures), which advocated and reinforced the results obtained during response surface interpretations. Intersecting curved and steep lines were indicative of the presence of interaction among studied CMVs. The desirability, as well as overlay plot, represented chromatographic conditions for obtaining optimum values of all the three CQAs. Relying on the above-obtained conditions the method was subjected to validation studies.

\section{Method validation studies}

\section{Specificity}

Forced degradation studies were performed with the dual intention of ascertaining method specificity as well as establishing stability behaviour of the drug. Visual evaluation of the chromatograms of stressed drug solutions advocated method specificity (Figure 4). The forced degradation experiments were carried out with an objective of obtaining degradation products with minimal use of chemicals along with a study protocol consuming less time. As a general start point based on prior knowledge and practice $0.1 \mathrm{M} \mathrm{HCl}, 0.1 \mathrm{M}$ $\mathrm{NaOH}, 3 \% \mathrm{H}_{2} \mathrm{O}_{2}, 80{ }^{\circ} \mathrm{C}$ temperature and UV light at $365 \mathrm{~nm}$ were chosen to stress the analyte. The chemical reagents were utilized at a very low volume of $500 \mu \mathrm{L}$ for a minimal time period of $30 \mathrm{~min}$. In the case of $\mathrm{UV}$ radiation, the exposure time was up to $3 \mathrm{~h}$ with the intent of providing sufficient exposure to UV light for generating degradation products. GLP degraded to a smaller extent in applied peroxide, thermal and photolytic conditions. However, extensive degradation was observed in the acid and alkali stress conditions. GLP was completely degraded upon exposure to $0.1 \mathrm{M}$ $\mathrm{NaOH}$. Hence, the strength of $\mathrm{NaOH}$ was reduced and a $0.01 \mathrm{M} \mathrm{NaOH}$ was used to assess stability. The GLP degraded more than $50 \%$ of its initial content indicating its susceptibility towards the new alkaline condition than compared to the acid condition. Two additional peaks were noticed at $5.0 \mathrm{~min}$ (Degradation product-I; DP-I) and $6.3 \mathrm{~min}$ (Degradation product-II; DP-II), in both the acid and alkaline stress conditions, which may be attributed to the possible break down of the ester linkage between $\alpha$-cyclopentyl mandelic acid and 3-hydroxy-1, 1-dimethylpyrrolidinium bromide during exposure to both acid and alkaline stress. Further, according to USP, the DP-I i.e. $\alpha$-cyclopentyl mandelic acid is an official related compound C of GLP (USP, 2017) which was generated during the stress studies of GLP. Moreover, the compendial monograph reports two separate procedures for determining the organic impurities of GLP and in both of them cyclopentyl mandelic acid is a reported related substance. Method specificity was found intact as both the degradation product peaks were well resolved from GLP peak (resolution $>5$ ) as well as among them (resolution $>3$ ). The analyte was found stable to exposure to UV light up to $3 \mathrm{~h}$ suggesting suitability of the process for regular routine use. The results endorsed the study intent (Table IV). 


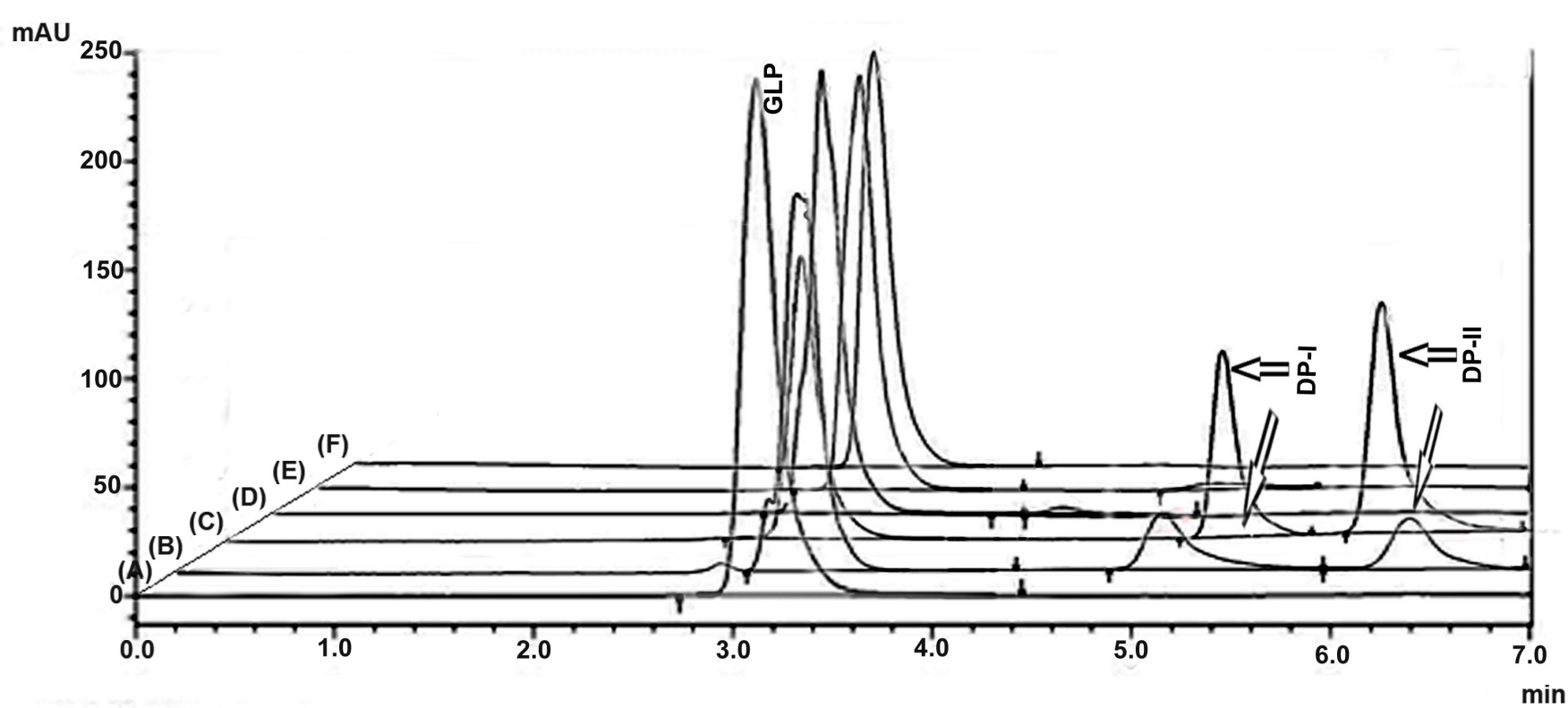

FIGURE 4 - Typical chromatograms depicting GLP pure drug (A) and after treatment with $0.1 \mathrm{M} \mathrm{HCl}$ (B), $0.01 \mathrm{M} \mathrm{NaOH}(\mathrm{C})$, $3 \% \mathrm{H}_{2} \mathrm{O}_{2}(\mathrm{D}), 80^{\circ} \mathrm{C}$ temperature (E) and UV light at $365 \mathrm{~nm}(\mathrm{~F})$.

Table IV - Results of forced degradation study

\begin{tabular}{|c|c|c|c|}
\hline Stress Condition & Time Period & Retention time(min) & Degradation/No Degradation \\
\hline $0.1 \mathrm{M} \mathrm{HCl}$ & $30 \mathrm{~min}$ & $\begin{array}{c}\text { Drug: } 3.189 \\
\text { Unknown DP-I: } 5.060 \\
\text { Unknown DP-II: } 6.360\end{array}$ & Degradation \\
\hline $0.01 \mathrm{M} \mathrm{NaOH}$ & $30 \mathrm{~min}$ & $\begin{array}{c}\text { Drug:3.154 } \\
\text { Unknown DP-I: } 5.078 \\
\text { Unknown DP-II: } 6.356\end{array}$ & Degradation \\
\hline $3 \% \mathrm{H}_{2} \mathrm{O}_{2}$ & $30 \mathrm{~min}$ & $\begin{array}{c}\text { Drug:3.164 } \\
\text { Unknown DP: } 4.464\end{array}$ & Degradation \\
\hline $80^{\circ} \mathrm{C}$ & $30 \mathrm{~min}$ & $\begin{array}{c}\text { Drug: } 3.148 \\
\text { Unknown DP: } 5.244\end{array}$ & Degradation \\
\hline UV light (365 nm) & $3 \mathrm{~h}$ & Drug: 3.129 & No Degradation \\
\hline
\end{tabular}


Linearity

The method was found linear over the concentration range of 5-250 $\mu \mathrm{g} \cdot \mathrm{mL}^{-1}\left(\mathrm{r}^{2}=0.999\right)$. Further, satisfactory results obtained through regression analysis and ANOVA of linearity data indicated the goodness of fit.

Accuracy

Satisfactory recoveries of GLP between 99.64$100.35 \%$, advocated for optimum method accuracy and reliability.

\section{Precision}

The precision study revealed acceptable values of $\% \operatorname{RSD}(<2 \%)$. The values were $0.63 \%, 0.74 \%$ and $0.37 \%$ for intraday, inter-day and system precision, respectively.

\section{Limit of detection (LOD) \& limit of quantitation (LOQ)}

The LOD and LOQ values were 2 and $5 \mu \mathrm{g} \cdot \mathrm{mL}^{-1}$, respectively.

\section{Analytical control strategy}

Preparation of control charts (Table V) helped to develop analytical control strategies. Reproducible results for CQAs were obtained by working within the analytical control space. The control space was defined to be within limits such as methanol proportion $( \pm 2 \%)$, the strength of TBAHS $( \pm 0.5 \mathrm{mM})$ and, flow rate $( \pm 0.1$ $\left.\mathrm{mL} \cdot \mathrm{min}^{-1}\right)$.

Table V - Results of control charts obtained for CQAs

\begin{tabular}{lccc}
\hline Parameter & $\begin{array}{c}\text { Retention } \\
\text { Time (min) }\end{array}$ & $\begin{array}{c}\text { Theoretical } \\
\text { Plate Count }\end{array}$ & $\begin{array}{c}\text { Symmetry } \\
\text { Factor }\end{array}$ \\
\hline Mean & 3.153 & 2738.88 & 1.626 \\
S.D. & 0.0012 & 6.614 & 0.0012 \\
R.S.D. (\%) & 0.038 & 0.241 & 0.073 \\
\hline
\end{tabular}

Table V - Results of control charts obtained for CQAs

\begin{tabular}{lccc}
\hline Parameter & $\begin{array}{c}\text { Retention } \\
\text { Time (min) }\end{array}$ & $\begin{array}{c}\text { Theoretical } \\
\text { Plate Count }\end{array}$ & $\begin{array}{c}\text { Symmetry } \\
\text { Factor }\end{array}$ \\
\hline LCL & 5.111 & 4227.57 & 1.381 \\
\cline { 2 - 4 } UCL & 5.114 & 4262.43 & 1.383 \\
\hline
\end{tabular}

\section{Assay of a commercial formulation}

The visual evaluation of chromatograms obtained for injections indicated method selectivity due to the non-interference of any of the formulation components (Figure 5). The mean $(n=3)$ content of GLP was found to be $98.33 \%$ ( $\mathrm{SD}= \pm 0.29$ ). Further, a visual comparison of chromatograms of standard GLP and GLP in injection preparation was carried out to establish method selectivity (Figure 6).

\section{CONCLUSIONS}

The present research explains the optimization and development of a UFLC method for estimating GLP in bulk and dosage forms. To achieve the objective a systematized analytical lifecycle management approach was followed. Utilizing the ALM approach, not only ensured increased method robustness but also presented an option for continuous improvement in the performance of CQAs. It helped to discover three CMVs and their effects on the CQAs. Based on the result of control charts, control strategies were outlined to obtain desired UFLC method performance. Overall, this analytical method is suitable and trustworthy for estimating GLP. Results of the validation study were found compliant with ICH guidelines. Forced degradation studies revealed that GLP is highly susceptible to the applied acid and alkaline stress conditions and produced two degradation products DP-I and DP-II, which calls for adequate preventive measures to be developed by the formulation scientist to protect therapeutic safety and efficacy of the pharmaceutical product. The forced degradation conditions were satisfactory as a known related compound $\mathrm{C}$ was generated as impurity implying appropriateness of the selected stress conditions. The optimum system suitability and satisfactory assay results depicting non-interference of the formulation 

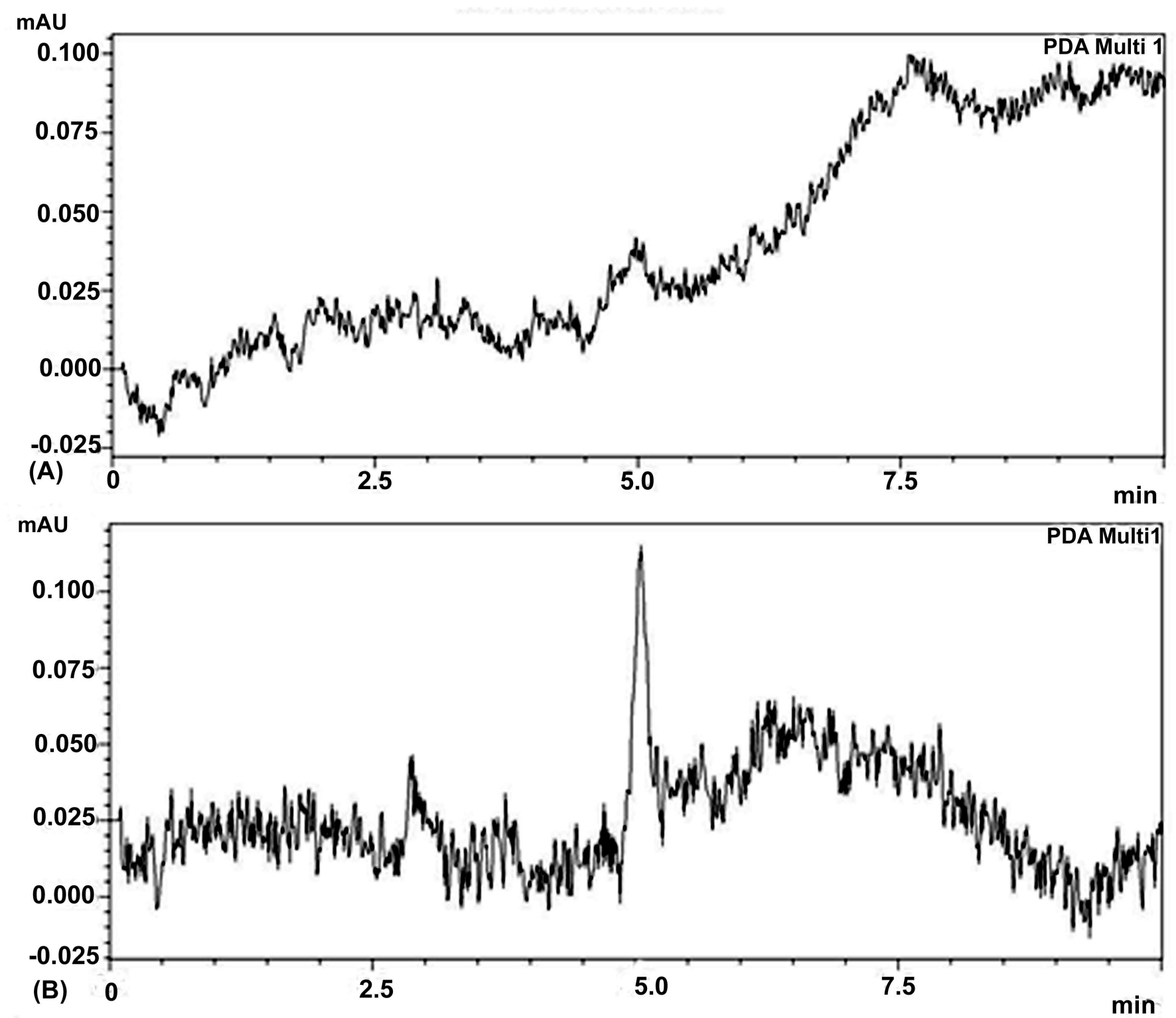

FIGURE 5 - Typical chromatograms depicting non-interference of formulation components at analytes retention time when blank mobile phase was injected with (A) hydrochloric acid and sodium hydroxide mixed solution, (B) benzyl alcohol solution. 
mAU

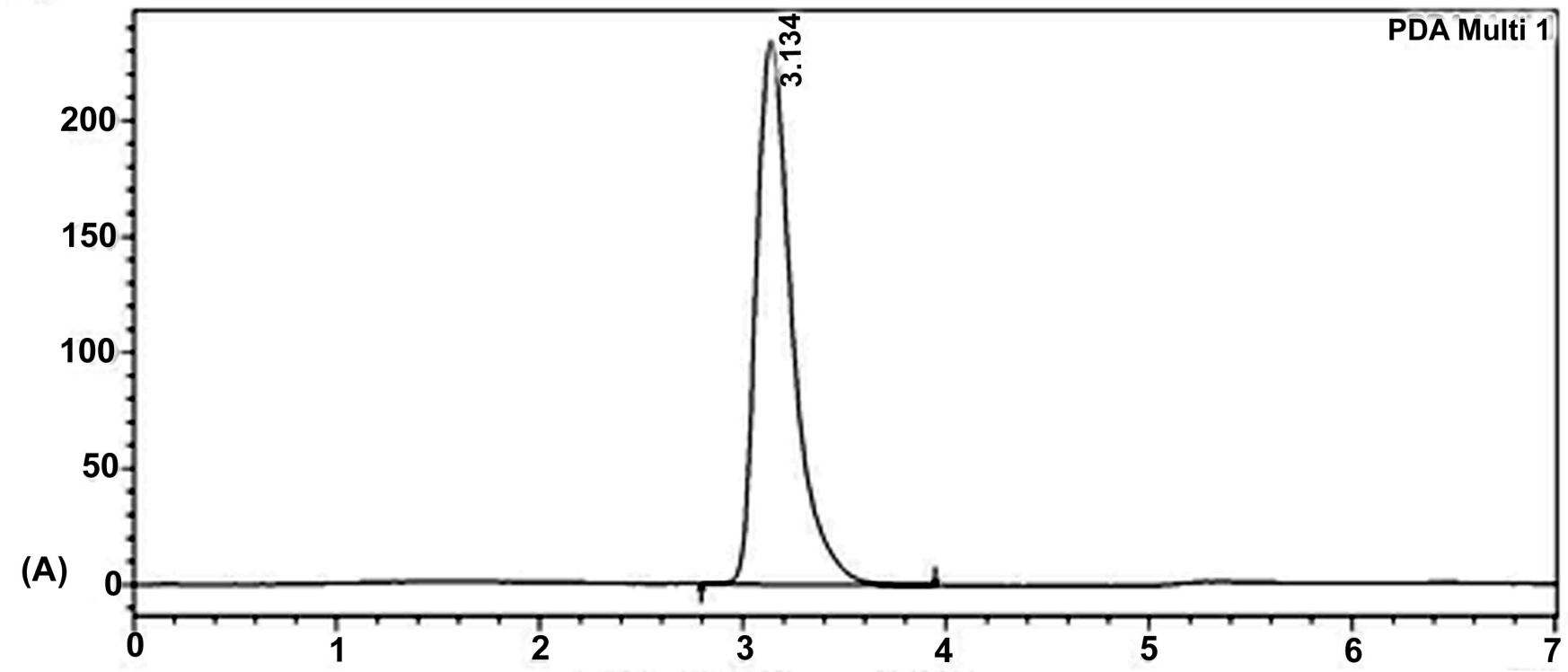

mAU

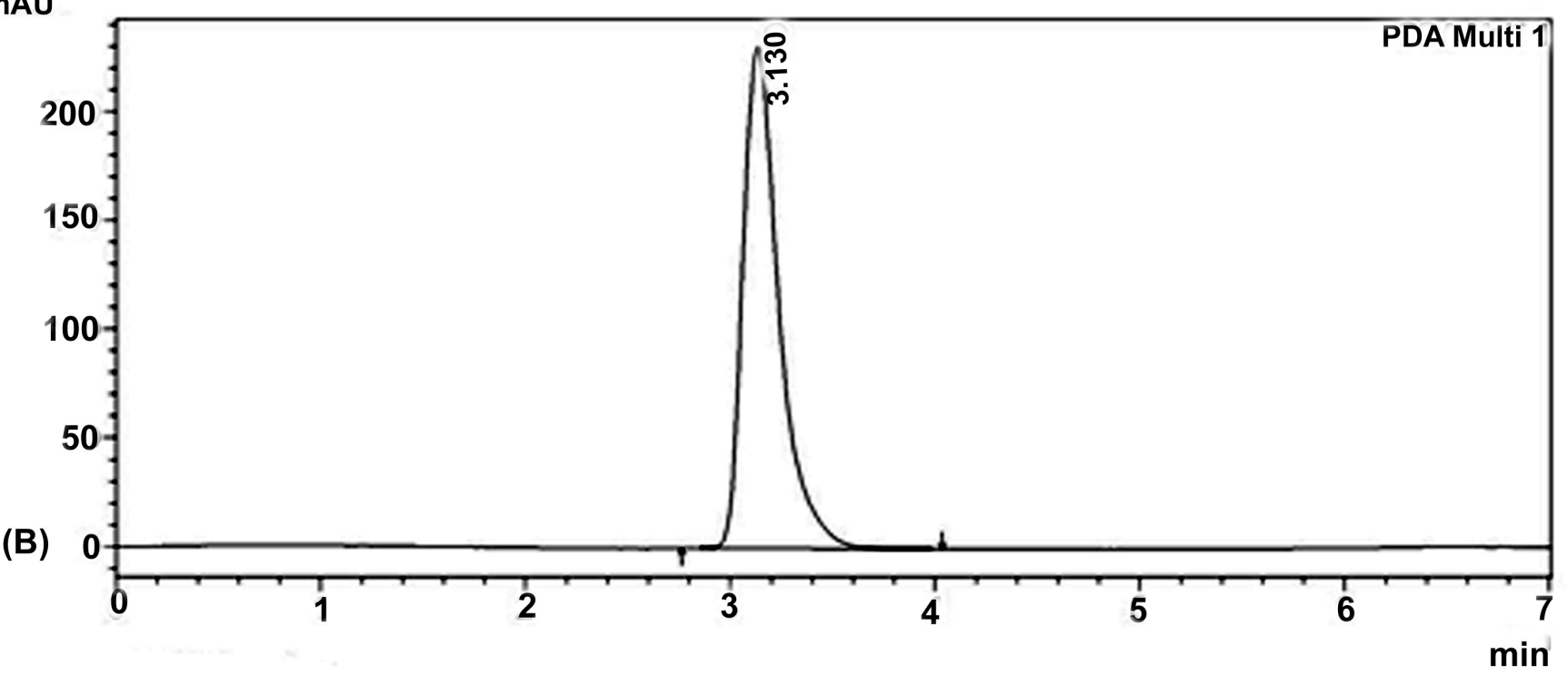

FIGURE 6 - Typical chromatograms of GLP standard drug (A) and GLP in injection formulation (B)

components advocated for the accomplishment of ATP. Hence, this method is acceptable for estimating GLP in bulk and injection formulation. Further, this method has the potential for determining GLP in biomatrixes.

\section{ACKNOWLEDGEMENTS}

The authors are thankful to Principal, Roland Institute of Pharmaceutical Sciences, Berhampur for providing the necessary research facilities.

\section{REFERENCES}

CMC Review of NDA 22-571. Application Number: 022571Orig1s000. Center for Drug Evaluation and Research. US FDA; 2010. [cited 2018 Feb 11] Available from: https://www.accessdata.fda.gov/drugsatfda_docs/ nda/2010/022571Orig1s000ChemR.pdf.

Deangelis NJ, Papariello GJ. Differential scanning calorimetry. Advantages and limitations for absolute purity determinations.J Pharm Sci. 1968; 57(11):1868-1873. 
Evatt ML. Oral glycopyrrolate for the treatment of chronic severe drooling caused by neurological disorders in children. Neuropsych Dis Treat.2011;7(1):543-547.

Gandala K, Pabba P, Akula G. RP-HPLC method development and validation for estimation of glycopyrrolate in bulk and tablet dosage forms. Asian J Pharm ClinRes. 2011; 4(4):p.37-40.

Glycopyrrolate. USP 40. Rockville: The United States Pharmacopeial Convention; 2017. p.4424.

Brennan WP, DiVito MP, Fyans RL, Gray AP. An overview of the calorimetric purity measurement. In: Blaine RL, Schoff CK, editor. Purity Determinations by Thermal Methods: A Symposium.Philadelphia: ASTM STP 838, American Society for Testing and Materials; 1984. p. 5-15.

International Conference on Harmonization. ICH. Validation of analytical procedures: text and methodology Q2(R1), Geneva: International Conference on Harmonization, 2005. [cited 2018 Feb 11] Available from: http://www.ich.org/fleadmin/Public_Web_ Site/ICH_Products/Guidelines/Quality/Q2_R1/Step4/Q2_ R1__Guideline.pdf.

International Conference on Harmonization. ICH. Impurities in new drug products Q3B (R2), Geneva: International Conference on Harmonization, 2006. [cited 2018 Feb 11] Available from: https://www.ich.org/fileadmin/Public_Web_ Site/ICH_Products/Guidelines/Quality/Q3B_R2/Step4/ Q3B_R2_Guideline.pdf

Kusuma J, Rao MVB, Rameshraju R. Development and validation of stability indicating RP-HPLC method for the estimation of glycopyrrolate and neostigmine in bulk and tablet dosage form. Int J Sci Engg Res. 2016;7(12)L1236-1242.

Lifecycle management of analytical procedures: method development, procedure performance, qualification, and procedure performance verification. Stimuli to the Revision Process: USP, 2013. [cited 2018 Feb 11]. Available from: http://www.ivtnetwork.com/article/lifecycle-management- analytical-procedures-method-development-procedureperformance-qualif- 0 .

Misra S, Arora V. Analytical Method Validation of HPLC Method for Assay of Anticholinergic Drug in Parenteral Formulation. IntJ Pharm Res Scho. 2016;5(4):101-104.

Nebiu D, Walter M, Lachmann B, Kopelent H, Noe CR. Determination of ( $\mathrm{R}, \mathrm{R})$-glycopyrronium bromide and its related impurities by ion-pair HPLC. Pharmazie.2007; 62(6):406-410.

O'Neil MJ, editor. The Merck Index, $14^{\text {th }}$ ed. New Jersey: Merck Research Laboratories, Merck \& Co., Inc., 2006.p.777.

Parr MK, Schmidt AH. Life cycle management of analytical methods. J Pharm Biomed Anal. 2018;147:506-517.

Panda SS, Ravi kumar BVV, Patel PK, Panda J. New RPUFLC method for estimation of xanthinol nicotinate in pharmaceutical dosage form. Chem Sci Trans. 2013a; 2(2):656-662.

Panda SS, Ravi kumar BVV, Mohanta G, Panda J. Reverse phase ultra fast liquid chromatography method for simultaneous estimation of citicoline sodium and piracetam in tablets. Int J Pharm Sci Nanotech.2013b; 6(1):1952-1957.

Panda SS, Ravi kumar BVV, Acharjya SK, Sahu K. RPUFLC method for estimation of propafenone in tablets. Int J Pharm Sci Nanotech. 2014;7(4):2671-2676.

Rumpler MJ, Sams RA, Colahan P. Validation of liquid chromatography tandem mass spectroscopy for determination of glycopyrrolate in horse plasma. J Anal Toxicol.2011;35(9):656-664.

van Dooren AA, Muller BW. Purity determinations of drugs with differential scanning calorimetry (DSC)-a critical review. Int J Pharm. 1984;20(3):217-233.

Received for publication on $06^{\text {st }}$ June 2018 Accepted for publication on $12^{\text {nd }}$ July 2019 